Universidad de La Salle

Ciencia Unisalle

Utopía - Working papers

Utopía

2020-12-01

\title{
Establecimiento y evaluación del potencial de Amblyseius herbicolus como depredador de mosca blanca (Bemisia tabaci)
}

Fredy Alexander Rodríguez Cruz

Universidad de La Salle, Bogotá

Juan Camilo Andrade Daza

Universidad de La Salle, Bogotá

Norelly Buesaquillo Samboní

Universidad de La Salle, Bogotá

Efrén Duván Gutiérrez Hernández

Universidad de La Salle, Bogotá

Julian Danilo Muñoz Imbachí

Universidad de La Salle, Bogotá

See next page for additional authors

Follow this and additional works at: https://ciencia.lasalle.edu.co/utopia_working_papers

\section{Recommended Citation}

Rodríguez Cruz, Fredy Alexander; Andrade Daza, Juan Camilo; Buesaquillo Samboní, Norelly; Gutiérrez Hernández, Efrén Duván; Muñoz Imbachí, Julian Danilo; and Velasco Samboní, Yucely, "Establecimiento y evaluación del potencial de Amblyseius herbicolus como depredador de mosca blanca (Bemisia tabaci)" (2020). Utopía - Working papers. 5.

https://ciencia.lasalle.edu.co/utopia_working_papers/5

This Documento de trabajo is brought to you for free and open access by the Utopía at Ciencia Unisalle. It has been accepted for inclusion in Utopía - Working papers by an authorized administrator of Ciencia Unisalle. For more information, please contact ciencia@lasalle.edu.co. 


\section{Autor}

Fredy Alexander Rodríguez Cruz, Juan Camilo Andrade Daza, Norelly Buesaquillo Samboní, Efrén Duván Gutiérrez Hernández, Julian Danilo Muñoz Imbachí, and Yucely Velasco Samboní 
https://doi.org/10.19052/wp.utopia.2020.1

\title{
Utopía | 10 \\ Working Papers \\ 2020
}

\section{Establecimiento y evaluación del potencial de Amblyseius herbicolus como depredador de mosca blanca (Bemisia tabaci)}

\author{
Fredy Alexander Rodríguez Cruz ${ }^{1}$, Juan Camilo Andrade Daza², Norelly Buesaquillo Samboní2, Efrén Duván Gutiérrez \\ Hernández ${ }^{2}$ Julián Danilo Muñoz Imbachí , Yucely Velasco Samboní2
}

\section{RESUMEN}

La mosca blanca (Bemisia tabaci) es un insecto altamente polífago, capaz de atacar más de 700 especies de vegetales, incluidos cultivos de importancia económica para el hombre. El control de esta plaga es realizado generalmente, por medio de la aplicación de insecticidas de síntesis química, con todos los problemas ambientales, sociales y de salud debido a su mal uso y abuso. Una alternativa a este tipo de control es el empleo del control biológico, donde se busca reestablecer la relaciona ecológica existente entre las poblaciones de artrópodos plaga y sus controladores naturales. El género Amblyseius sp. se destaca dentro de la familia de ácaros depredadores Phytoseiidae, ha sido evaluado en diversos ensayos en laboratorio y en campo, demostrando una buena eficacia en el control de varias plagas de artrópodos. Poblaciones naturales de este ácaro fueron registradas en asocio a poblaciones de mosca blanca en el cultivo de yuca en el Campus Utopía. Inicialmente se estableció en laboratorio una cría de Amblyseius herbicolus. En laboratorio fue evaluado el potencial de esta especie depredadora para el control de mosca blanca. Los resultados indican que Amblyseius herbicolus tiene la habilidad de alimentarse de huevos y ninfas de plaga cuando se le ofrecieron separados o mezclados, y que estos poseen los requerimientos nutricionales que le permiten a la hembra del depredador la oviposición. Los resultados plantean una nueva estrategia de control a ser investigada más profundamente para el control de esta importante plaga agrícola.

Palabras clave: Control biológico, Phytoseiidae, Manihot esculenta, Ecología

\section{Establishment and evaluation of the potential of Amblyseius herbicolus as predator of whitefly (Bemisia tabaci)}

\begin{abstract}
The whitefly (Bemisia tabaci) is a highly polyphagous insect, capable of attacking more than 700 species of plants, including crops of economic importance to man. The control of this pest is generally carried out, through the application of chemical synthesis insecticides, with all the environmental, social and health problems due to its misuse and abuse. An alternative to this type of control is the biological control, strategy which seeks to reestablish the ecological relationship between the populations of plague arthropods and their natural controllers. The genus Amblyseius sp. It
\end{abstract}

${ }^{1}$ Docente del programa de ingeniería Agronómica de la Universidad de La Salle

${ }^{2}$ Estudiante de ingeniería Agronómica de la Universidad de la Salle 


\section{Utopía | \\ Working Papers \\ 2020}

stands out within the family of predatory mites Phytoseiidae, it has been evaluated in various laboratory and field trials, demonstrating good efficacy in the control of several arthropod pests. Natural populations of this mite were recorded in association with whitefly populations in the cassava crop at the Utopia Campus. Initially, a rearing Amblyseius herbicolus was established in the laboratory. In the laboratory, the potential of this predatory species to control whitefly was evaluated. The results indicate that Amblyseius herbicolus feed on eggs and nymphs of the pest, offered single or mixed, and that they possess the nutritional requirements that allow the female predator to oviposit. The results propose a new control strategy to be investigated more deeply for the control of this important agricultural pest.

Keywords: Biological control, Phytoseiidae, Manihot esculenta, Ecology

\section{INTRODUCCIÓN}

La mosca blanca (Bemisia tabaci Gennadius) es una plaga de importancia económica en el cultivo de yuca (Manihot esculenta Crantz), pues es el principal vector del virus africano de la yuca; además esta plaga produce daños a la planta de forma directa, al ser un insecto chupador de savia e indirectamente, al secretar sustancias azucaradas que sirven como sustrato para el desarrollo de fumagina, la cual es un hongo que cubre totalmente la hoja, impidiendo la entrada de luz que puede aprovechar la planta para la fotosíntesis (Bellotti et al., 2007).

La aplicación de productos químicos es el principal método de control de este insecto. Sin embargo, el abuso de este tipo de productos genera contaminación al medio ambiente y un riesgo constante de la adquisición de resistencia a estos por parte de la plaga. Adicionalmente, el desconocimiento de los agricultores sobre la existencia de otros medios de control de plagas puede llevar a un uso indiscriminado de productos sintéticos causando la eliminación de enemigos naturales propios de la plaga.

Una alternativa al control químico es la promoción de la aplicación de productos biológicos o el uso de enemigos naturales, con el propósito de reducir los índices de las poblaciones de mosca blanca y la contaminación ambiental (Bellotti et al., 2007).

La familia Phytoseiidae posee varias especies de ácaros depredadores, los cuales atacan diversas plagas como ácaros fitófagos, trips o moscas blancas (Ali, 1998; McMurtry et al., 1997, Nomikou et al, 2001). Estos ácaros son capaces de trasladarse dentro de la planta y entre plantas en búsqueda de su alimento (Gotoh et al, 2004, Van Lenteren, 2003, Nicholis, 2008). Dentro de la familia se destaca el género Amblyseius (Berlese, 1914). 
https://doi.org/10.19052/wp.utopia.2020.1

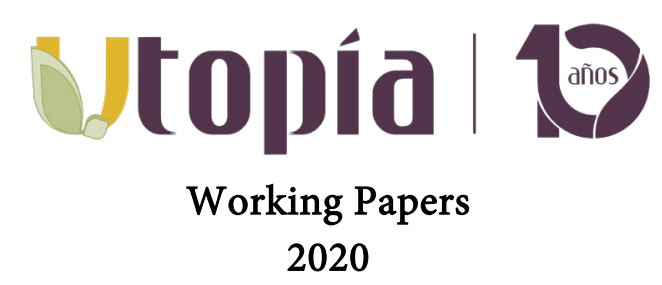

El género Amblyseius pertenece a la clasificación denominada como depredadores generalistas, los cuales se alimentan de otros ácaros de las familias Acaridae, Pyroglyphidae, Tetranychidae, Tenuipalpidae y Tydeidae, así como de trips, moscas blancas, cochinillas e inclusive nemátodos; pero también pueden alimentarse y reproducirse bien en polen de diferentes especies vegetales. Igualmente, pueden utilizar exudados de plantas y mielato como alimento en ausencia de presas o como alimento complementario, lo que aumenta su capacidad reproductiva cuando hay presas (McMurtry y Croft 1997, McMurtry et al., 2013).

El objetivo de la investigación fue establecer crías y evaluar el potencial de Amblyseius herbicolus (Chant) como controlador biológico de mosca blanca (Bemisia tabaci Gennadius) en condiciones de laboratorio. La investigación se llevó a cabo en el laboratorio de Entomología del Campus Utopía de la Universidad de La Salle ubicada en el km 12 vía Matepantano en Yopal-Casanare.

\section{METODOLOGÍA}

La investigación se llevó a cabo en el laboratorio de Entomología del Campus Utopía de la Universidad de La Salle ubicada en el km 12 vía Matepantano en Yopal-Casanare.

Cria: inicialmente se realizaron colectas en hojas de yuca, variedad MCol 2737. Varios especímenes del ácaro depredador fueron montados en lámina de microscopia con ayuda de medio Hoyer para su identificación, indicando tratarse de la especie Amblyseius herbicolus. Los especímenes colectados fueron dispuestos en arenas de cría, las cuales fueron elaboradas de una lámina $(25 \times 12 \mathrm{~cm})$ de PVC dispuesta sobre un bloque de espuma ( 28 x 15x $3 \mathrm{~cm}$ ); alrededor de los bordes de la hoja de PVC se dispuso papel Kleenex ${ }^{\oplus}$, con el doble fin de servir como fuente de agua y barrera física para impedir el escape de los depredadores. Este sistema se ubicó dentro de una bandeja plástica $(30$ x 18 x $5 \mathrm{~cm}$ ), la cual contenía agua y jabón, para evitar la contaminación con otros organismos. 
https://doi.org/10.19052/wp.utopia.2020.1

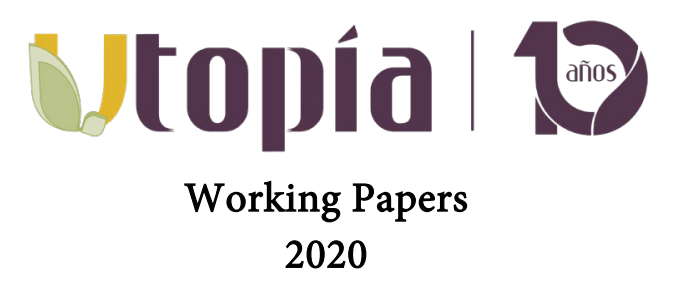

Como fuente de alimento para el predador se utilizó polen de abeja comercial que fue proporcionado directamente en la lámina de PVC; como sitio de refugio y oviposición fue dispuesto un trozo de PVC $(2 \times 2$ cm), el cual fue doblado a la mitad colocándose un trozo de algodón debajo de él.

Ante las dificultades para el establecimiento definitivo de la especie, otras colectas fueron realizadas tanto en hojas de yuca, como en hojas de café (Coffea arabica) para extraerlo de estas y reforzar las arenas de cría, llevando a su conservación por un mayor tiempo y establecimiento definitivo.

\section{Evaluación de la capacidad de depredación}

La evaluación se llevó a cabo en discos de hoja de girasol (Helianthus annuus) ( $\varnothing=45 \mathrm{~mm}$ ), los cuales se mostraron mucho más adecuados que los realizados con hojas de yuca, pues estos mostraban un rápido proceso de descomposición. Los discos de hoja de girasol fueron dispuestos sobre una capa de PDA (Potato Dextrosa Agar) de un centímetro de altura.

Para la evaluación de la capacidad de depredación de $A$. herbicolus, se efectuaron cuatro tratamientos con cinco repeticiones cada uno. Los tratamientos fueron:

Tratamiento 0: Testigo (1 depredador Amblyseius herbicolus, sin alimento).

Tratamiento 1: (30 huevos de Bemisia tabaci y 1 depredador Amblyseius herbicolus).

Tratamiento 2: (15 ninfas de Bemisia tabaci y 1 depredador Amblyseius herbicolus).

Tratamiento 3: (20 huevos y 10 ninfas de Bemisia tabaci y 1 depredador Amblyseius herbicolus).

Los diferentes estadios de la plaga se recolectaron en hojas de yuca con presencia de mosca blanca y con ayuda de un pincel fueron transferidos a los discos de hoja; las cantidades de cada estadio de la plaga fueron generadas a partir de pruebas piloto. 


\section{Utopía | 10 \\ Working Papers \\ 2020}

Las variables evaluadas fueron: No. de presas de B. tabaci consumidas y No. de huevos producidos por el depredador. La toma de datos se llevó a cabo durante 3 días. Los datos correspondientes al primer día de evaluación no fueron considerados en el análisis estadístico debido a los efectos de la dieta previa.

Los datos del día dos y tres de evaluación fueron sumados y analizados empleando Modelos Lineales Generalizados (GML, por sus siglas en ingles), con distribución de errores tipo Poisson. Los contrastes entre las diferentes dietas fueron determinados con la prueba de hipótesis general lineal (función glht del paquete lsmeans en R, Lenth 2016). Los análisis fueron realizados en el software estadístico R 3.4 (R Development Core Team, 2018).

\section{RESULTADOS}

Hubo diferencia estadística entre el número de individuos depredados en las diferentes dietas (GLM, $X^{2}=33,38$; d.f $=3 ; \mathrm{p}<0,001)$ (figura 1$)$.

Respecto a la oviposición, también hubo diferencia estadística en el número de huevos según la dieta (GLM, $X^{2}$ $=9,58 ;$ d.f $=3 ; \mathrm{p}<0,02)$ (figura 2 ).

\section{DISCUSIÓN}

El tratamiento 3 (Mezcla: huevo/ninfa) presentó un mayor consumo por parte del depredador. Según Rodríguez et al. (2017), el predador manifiesta un comportamiento alimenticio visible de la mezcla entre huevos y ninfas. Posteriormente su alimentación va seguida de los huevos ofrecidos independientemente. 


\section{Utopía | \\ Working Papers \\ 2020}

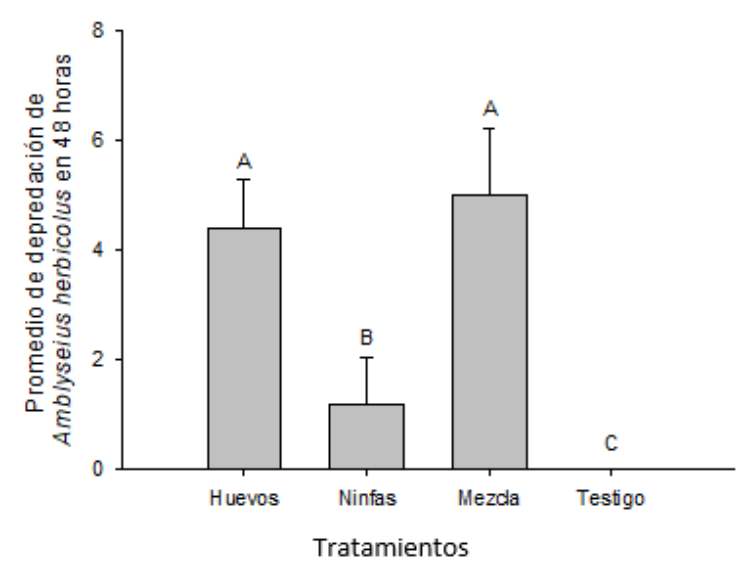

Figura 1. Cantidad de individuos predados ( $\pm \mathrm{EE})$ por Amblyseius herbicolus en cada uno de los tratamientos evaluados.

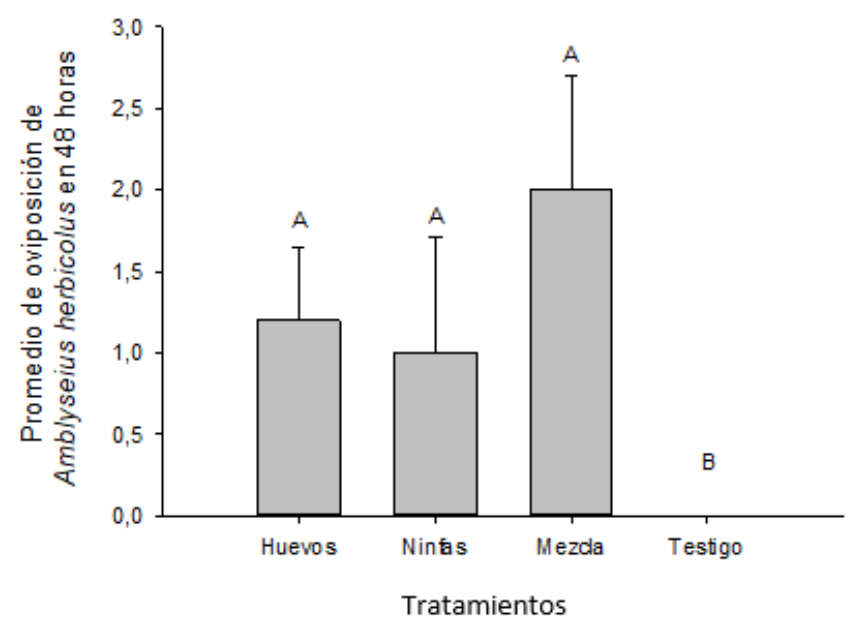

Figura 2. Producción de huevos promedio ( \pm EE) de Amblyseius herbicolus en cada una de las dietas evaluadas.

No hubo diferencia significativa entre los tratamientos donde se ofrecieron estadios de la plaga, mientras que el testigo fue diferente de las dietas ofrecidas. Según Rodríguez et al. (2017), el predador es capaz de ovipositar al alimentarse de las diferentes dietas (tratamientos). Sin embargo, el testigo al no ofrecer ningún recurso al 


\section{Utopía | \\ Working Papers \\ 2020}

depredador resulta en la incapacidad de la hembra para ovipositar. Entretanto, la hembra del depredador es capaz de aprovechar eficientemente los estadios de la mosca blanca que fueron ofrecidos.

Forero et al. (2008) mencionan que el género Amblyseius sp., en condiciones de laboratorio e invernadero, es capaz de consumir más larvas y ninfas que huevos Tetranychus urticae. Entretanto, Schausberger et al., (2001), afirman que el ácaro depredador muestra hábito de preferencia por estados inmaduros inmóviles de $B$. tabaci. Pero contrastando la preferencia en ácaros fitófagos como Tetranychus sp., donde hay una preferencia hacia los estadios inmaduros móviles.

Cavalcante et al. (2015), al evaluar cuatro especies diferentes de Phytoseiidae (A. limonicus, A. herbicolus, $A$. largoensis y $A$. tamatavensis) sobre mosca blanca, encontraron que todos los depredadores se alimentaron de huevos de la plaga. Sin embargo, A. herbicolus, presentó niveles más altos de depredación. Esto se corrobora con los resultados obtenidos en la presente investigación.

Holling, (1959), citado por Huffaker y Gutiérrez, (1999) afirma que Amblyseius sp. aumenta su tasa de consumo al aumentar la densidad de las presas. Sin embargo, va desacelerando gradualmente hasta llegar a un punto donde esta tasa es constante, independiente de la densidad de presa, es decir que, por su hábito generalista, el ácaro buscará otra opción de alimento, variando su dieta.

Por otra parte, se demostró que el fitoseído, en general, si tuvo eficacia en predar B. tabaci en sus estadios inmaduros (huevo/ninfa). Calvo (2011), manifiesta que el ácaro en condiciones de laboratorio fue capaz de regular eficazmente poblaciones de mosca blanca en sus primeros estadios en plantas de pepino.

Amblyseius herbicolus posee reproducción asexual mediante partenogénesis telitoquia. Según Shipp y Whitfield, (1991), las hembras consumen mayor cantidad de alimento, debido a que poseen un gasto de energía extra relacionado con el desarrollo de los huevos.

Por último, Estos resultados indican que experimentos en condiciones semejantes a las de campo son necesarios para continuar con la evaluación del depredador y dilucidar su verdadero potencial como enemigo natural de esta importante plaga en las condiciones de Yopal-Casanare. 


\section{Utopía | \\ Working Papers \\ 2020}

\section{CONCLUSIONES}

Amblyseius herbicolus consiguió alimentarse de los estadios de mosca blanca ofrecidos, huevos y ninfas, tanto aisladamente como en mezcla. Los estadios depredados indican que son nutricionalmente adecuados, pues su consumo resulto en oviposición de las hembras. Sin embargo, parece ser que la hembra del depredador obtiene mayor beneficio al alimentarse de la mezcla de los estadios que al utilizarlos independientemente, pues si bien no hubo diferencia estadística entre la oviposición, el número de huevos depositados al ingerir los estadios en mezcla fue superior a la oviposición generada al alimentarse de solo un estadio.

A pesar de ser un experimento simple, los resultados indican un potencial de control de poblaciones de mosca blanca por parte del depredador. Sin embargo, son necesarios nuevos experimentos en situaciones más próximas a las encontradas en campo para corroborar este potencial encontrado en laboratorio.

\section{BIBLIOGRAFÍA}

Belda, J. E., Calvo, J. (2006). Eficacia de Amblyseius swirskii Athias-Henriot (Acari: Phytoseiidae) en el control biológico de Bemisia tabaci (Genn.) (Hom: Aleyrodidae) y Frankliniella occidentalis (Pergande) (Thys: Thripidae) en pimiento en condiciones de semicampo. Bol San Veg Plagas, 32(3), 283-296.

Bellotti, A. C., Arias, V., Herrera Fernández, C. J., Holguín, A., María, C. (2007). Manejo integrado de moscas blancas asociadas al cultivo de la yuca. CIAT.

Calvo, F. J. (2011). Implicaciones de Amblyseius swirskii Athias-Henriot (Acari: Phytoseiidae) en la mejora del control biológico de Bemisia tabaci Gennadius (Hemiptera: Aleyrodidae) en el cultivo de pimiento en invernadero.

Cavalcante, A. C. C., Dos Santos, V. L. V., Rossi, L. C., Moraes, G. J. D. (2015). Potential of five Brazilian populations of Phytoseiidae (Acari) for the biological control of Bemisia tabaci (Insecta: Hemiptera). Journal of economic entomology, 108(1), 29-33. 
https://doi.org/10.19052/wp.utopia.2020.1

\section{Utopía | \\ Working Papers \\ 2020}

Forero, G., Rodríguez, M., Cantor, F., Rodríguez, D., Cure, J. R. (2008). Criteria for the management of Tetranychus Urticae Koch (Acari: Tetranychidae) with the predator mite Amblyseius (Neoseiulus) sp. (Acari: Phytoseiidae) on rose culture. Agronomía Colombiana, 26(1), 78-86.

Huffaker, C., Gutiérrez, A. (1999). Entomología ecológica. Nueva York, John Wiley \& Sons, 756p.

McMurtry, J. A., Croft, B. A. (1997). Life-styles of Phytoseiidae mites and their roles in biological control. Annual review of entomology, 42(1), 291-321.

McMurtry, J. A., Moraes, G. J. D., Sourassou, N. F. (2013). Revision of the lifestyles of phytoseiid mites (Acari: Phytoseiidae) and implications for biological control strategies.

Moreno, I. P., Mancebón, V. S. M. (2011). Importancia y uso de los ácaros fitoseídos (Acari, Phytoseiidae) en el manejo agroecológico de plagas. In Manejo agroecológico de sistemas (pp. 69-92). Benemérita Universidad Autónoma de Puebla.

Nicholls, C. I. (2008). Control biológico de insectos: un enfoque agroecológico. Universidad de Antioquia.

Nomikou, M., Janssen, A., Schraag, R., Sabelis, M. W. (2001). Phytoseiidae predators as potential biological control agents for Bemisia tabaci. Experimental \& Applied Acarology, 25(4), 271-291.

Rodríguez-Cruz, F. A., Janssen, A., Pallini, A., Duarte, M. V. A., Pinto, C. M. F., Venzon, M. (2017). Two predatory mite species as potential control agents of broad mites. BioControl, 62(4), 505-513.

Schausberger, P., A. Walzer, A. 2001. Combined versus single species release of predaceous mites predatorpredator interactions and pest suppression. Biol. Control 20, 269-278.

Shipp, J.L., Whitfield, GH (1991). Respuesta funcional del ácaro predatorio, Amblyseius cucumeris (Acari: Phytoseiidae), en los trips de flores occidentales, Frankliniella occidentalis (Thysanoptera: Thripidae). Entomología ambiental, 20(2), 694-699. 


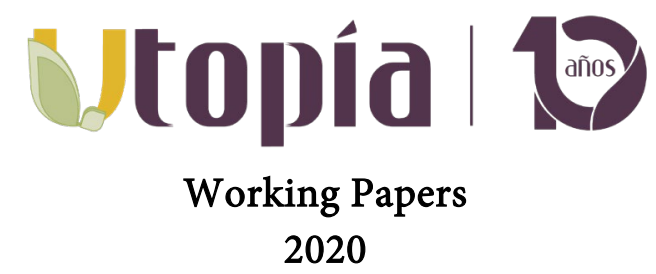

Vinasco, A., Soto, G., Vallejo, E., Fernando, L. (2014). Requerimientos térmicos para el desarrollo de Amblyseius sp. (Acari: Phytoseiidae). Boletín Científico. Centro de Museos. Museo de Historia Natural, 18(2), 61-66. 